\title{
The Effectivity of Anodal Transcranial Direct Current Stimulation in Enhancing Wrist Extensor Muscle Activation After Stroke: A Randomized Trial
}

\author{
Anindya K Zahra ${ }^{a}$, Meisy Andriana ${ }^{\mathrm{a} a^{*}}$, Lydia Arfianti ${ }^{a}$, I Putu Alit Pawana ${ }^{a}$, \\ Damayanti Tinduh ${ }^{a}$ \\ ${ }^{\mathrm{a}}$ meisy.andriana@fk.unair.ac.id \\ ${ }^{a}$ Physical Medicine and Rehabilitation Department, Faculty of Medicine Universitas Airlangga, Dr. Soetomo Academic General \\ Hospital, Surabaya, Indonesia
}

\begin{abstract}
Background: Disability in stroke patients is frequently due to upper limb motor dysfunction, in which wrist extension control plays a major role. Beneficial effects of anodal transcranial direct current stimulation (tDCS) on motor recovery after stroke have been previously reported, but data regarding its effect on wrist extensor muscle activity is limited.

Objective: To investigate whether anodal tDCS has an effect on wrist extensor activation in post-stroke patients.

Methods: Ten patients suffering from first ischemic stroke were randomized to either an experimental group (anodal tDCS + occupational therapy) or a control group (occupational therapy only). Anodal tDCS over ipsilesional primary motor cortex (M1) was delivered with $2 \mathrm{~mA}$ intensity for 20 minutes. Evaluations of wrist extensor muscle activation during isometric contraction using surface electromyography were performed before and after five days of intervention.

Results: Within-group comparison in the experimental and control groups; and between-group comparison showed no significant difference in wrist extension control before and after intervention $(p=0.446, p=0.764$, and $p=0,917$, respectively).

Conclusions: Five sessions of anodal tDCS showed no significant effect on wrist extensor activation in post-stroke patients. Further studies with a larger sample size and a longer period of intervention are necessary to clarify the effects of tDCS on wrist extension control in stroke patients.
\end{abstract}

Keywords: rehabilitation; transcranial direct current stimulation; stroke; surface electromyography

\section{Introduction}

Stroke is considered as one of the most disabling diseases (Feigin et al., 2017). Stroke prevalence is estimated to reach 77 million people in 2030 (Strong et al., 2007). Disability in stroke patients is frequently due to upper limb motor dysfunction, in which wrist extension control plays a major role (Cauraugh et al., 2000; Hunter \& Crome, 2002). Upper limb motor recovery after stroke is still unsatisfactory, regardless of rehabilitation (Barecca et al., 2003). Wrist extension control is important for performing activity of daily living (ADL) and affects dexterity and grip strength (Neumann, 2010; Souza et al. 2017). Yet it is one of the movements which is most difficult to achieve recovery after a stroke (Cauraugh et al., 2000).

Non-invasive brain stimulation techniques, such as Repetitive Transcranial Magnetic Stimulation (rTMS) and Transcranial Direct Current Stimulation (tDCS), are recommended adjunctive therapies in a multiple systematic review by Hatem et al. (2016) to improve the effectiveness of stroke rehabilitation in achieving upper limb motor recovery. Transcranial direct current stimulation is more practical, and inexpensive compared to rTMS (Klomjai et al., 2015).

Transcranial direct current stimulation can help optimize recovery through cortical excitability modulation (Schlaug et al., 2008; Hatem et al., 2016). There are several application techniques of tDCS, namely 1) anodal 
tDCS which increases cortical excitability; 2) cathodal tDCS which decreases cortical excitability; and 3) bilateral or dual tDCS which combines anodal and cathodal stimulation at the same time (Schlaug et al., 2008).

Anodal tDCS has been shown to be beneficial in motor rehabilitation following a stroke. The use of anodal tDCS in conjunction with functional task training enhanced hand dexterity, grip strength, and the upper extremity Fugl Meyer Assessment score (Cha et al., 2014). Regarding tDCS effect on wrist extensor muscle activity after stroke, only a few studies have been reported. A previous study conducted by Shaheiwola et al., (2018) showed that the combination of bilateral tDCS and Functional Electrical Stimulation (FES) may increase the activity of extensor carpi radialis longus than FES alone. While that study utilized a bilateral tDCS montage, this study used anodal tDCS since a meta-analysis study found that using tDCS with anodal stimulation to the lesioned M1 was significantly advantageous for the restoration of upper extremity motor function in stroke patients. Occupational therapy was employed as the standard therapy in this study instead of FES. In this investigation, the electrode placement was not specific to the ECRL, but rather quasi-specific to the wrist extensor muscle group. To the author's knowledge, this is the first randomized controlled trial conducted in Indonesia which investigates the effect of anodal tDCS on wrist extensor activity in stroke patients.

\section{Materials and Methods}

\subsection{Protocol}

A randomized controlled trial was performed to determine whether the use of anodal tDCS could improve wrist extensor activation in post-stroke patients. Ischemic stroke patients in our outpatient clinic were evaluated with regard to the inclusion/exclusion criteria reported in the following section (Participants). Patients were randomized into 2 groups. The experimental group received anodal tDCS and occupational therapy, while the control group (CG) received only occupational therapy. Occupational therapy sessions were performed immediately after anodal tDCS in the experimental group (EG). Interventions were carried out for five consecutive days. Evaluations of wrist extensor muscle activation using surface electromyography were performed prior to the intervention period and soon thereafter. This protocol was approved by the independent ethics committee of our hospital with the number 1957/KEPK/IV/2020, and all participants provided written informed consent.

\subsection{Participants}

Ten ischaemic stroke patients were recruited for this study. The inclusion criteria were as follows: (1) 30 to 70 years of age; (2) first ischemic stroke with onset of 2 weeks-12 months; (3) affected upper extremity strength scale of 2-4 (Medical Research Council scale); (4) absence of ROM limitation of the affected upper extremity; (5) able to understand instructions; (6) absence of cognitive disturbance; and (7) signed informed consent. The exclusion criteria were as follows: (1) severe spasticity on the affected upper extremity (MAS $\geq 3$ ); (2) pain on the affected upper extremity (pain scale $\geq 4$ ); (3) hemineglect; (4) apraxia; (5) hemianopsia; (6) metal implant in the head region; (7) pacemaker; (8) severe cardiorespiratory problems; (9) history of seizure or epilepsy; (10) existence of skin lesion on the stimulation area; (11) electrode hypersensitivity. All enrolled patients gave written informed consent prior to the initiation of the experimental protocol.

\subsection{Transcranial Direct Current Stimulation and Rehabilitation Procedures}

Participants in the intervention group received anodal tDCS for 20 minutes at intensity of $2 \mathrm{~mA}$, which was preceded by a gradual increase in intensity at the start of the session (ramp-up) and a gradual decrease at the end of the session (ramp-down) 30 seconds each. Anodal tDCS was delivered through two saline-soaked electrodes $\left(25 \mathrm{~cm}^{2}\right)$ using a constant current stimulator (ActivaDose II, Caputron, NY, USA). The anode was 
placed over the affected primary motor cortex or M1 (C3/C4 according to the International 10-20 EEG system), while the cathode was placed over the contralateral supraorbital region (Fp1/Fp2 according to the International 10-20 EEG system). Stimulation was performed immediately prior to the occupational therapy session.

Occupational therapy sessions following brain stimulation were part of standard hand rehabilitation program in our outpatient clinic, which followed the occupational performance model (Lee et al., 2015). In the present study, occupational therapy lasted 30 minutes for each session. Training sessions commenced with a five-minute warm-up consisting of active or active assistive upper limb ROM activities, sensory stimulation, and spastic inhibition if needed. Enabling activities were performed to promote sensorimotor integration and functional movement with task-dependent principles, including: reaching, grasping and releasing, and object manipulation. The intensity and type of exercises were tailored based on the patient's residual abilities.

\subsection{Outcome Measures (surface electromyography)}

For surface electromyography (sEMG) assessment, subjects were seated on a chair with their elbow in $90^{\circ}$ flexion and their wrist in $30^{\circ}$ extension. Wrist extensor muscle activity on the affected hand was recorded by sEMG (Medspec 4000, Megatronics, Finland) using self-adhesive 20-mm diameter $\mathrm{Ag}-\mathrm{AgCl}$ electrodes. The electrodes were positioned $3 \mathrm{~cm}$ apart over the skin (previously cleaned with alcohol) in the region of the extensor digitorum muscle, following the guidelines of the sEMG for the Non-invasive Assessment of Muscles (SENIAM) (Hermens et al., 2000). The assessment of wrist extensor activity in this study was quasispecific, which was accomplished by placing a surface electrode on top of the extensor digitorum muscle in accordance with Cram et al. (2011) recommendations, which allows signal measurements from the extensor digitorum, extensor carpi radialis, and extensor carpi ulnaris muscles (Cram et al., 2011). The extensor digitorum, although being a secondary muscle, can generate significant wrist extension torque (Neumann, 2010). It is also larger than other wrist extensor muscles and covers the majority of the forearm's posterior surface (Moore et al., 2010).

Signals were recorded at a sampling rate of $600 \mathrm{~Hz}$, amplified, and band-passed filtered using the data acquisition system Motus 32 software (Peak Performance Technologies, Englewood, USA). For data acquisition, participants performed isometric contraction of the wrist extensor against resistance for 5 seconds following a verbal command. Only the data in between 3 seconds was processed to avoid the influence of the transition motion state on sEMG assessment (Shaheiwola et al., 2018). The assessments were repeated three times, with two-to-three minutes of rest between each reading. Quantification of sEMG data using root mean square (RMS) was expressed in miliVolts. The best of three reading results were analyzed.

\subsection{Statistical Analysis}

Study outcomes were changes in wrist extensor muscle activation (root mean square) before and after intervention. Statistical analyses were performed with IBM SPSS Statistics for Macintosh, Version 26.0. Intra-group analysis of both groups used a paired $t$ test. Changes in RMS before and after intervention between the experimental and control group were analysed using the Mann-Whitney test. A $p$-value of $<0.05$ was accepted as significant.

\section{Results}

Ten eligible individuals with post ischemic stroke ( 7 women and 3 men; mean age 51 years) were recruited, provided informed consent, and initiated the study. They were randomized into either the Experimental Group (EG) or the Control Group (CG). All subjects were right-handed dominant. There were no significant characteristic differences between groups. Subject characteristics are provided in Table 1. 
Table 1. Subject characteristics

\begin{tabular}{lccc}
\multicolumn{1}{c}{ Characteristics } & $\begin{array}{c}\mathrm{EG}(\mathrm{n}=5) \\
\text { Mean } \pm \text { SD } \\
\text { Proportion (\%) }\end{array}$ & $\begin{array}{c}\mathrm{CG}(\mathrm{n}=5) \\
\text { Mean } \pm \text { SD } \\
\text { Proportion (\%) }\end{array}$ & $p$ value \\
\hline Age $^{\mathrm{a}}$ (years) & $50.00 \pm 13.84$ & $52.00 \pm 3.08$ & 0.760 \\
Stroke onset $^{\mathrm{a}}$ (months) & $7.00 \pm 4.06$ & $9.40 \pm 2.61$ & 0.299 \\
Sex $^{\mathrm{b} \text { Female }}$ & $4 / 5(80 \%)$ & $3 / 5(60 \%)$ & 1.000 \\
Affected Side $^{\mathrm{b}}$ & & & \\
$\quad$ Left hemiparesis (non-dominant) & $3 / 5(60 \%)$ & $3 / 5(60 \%)$ & 1.000 \\
Muscle strength (mMRC) & & & \\
$-\quad 2$ & $2 / 5(40 \%)$ & $2 / 5(40 \%)$ & 0.760 \\
$-\quad 3$ & $1 / 5(20 \%)$ & $2 / 5(40 \%)$ & \\
$-\quad 4$ & $2 / 5(40 \%)$ & $1 / 5(20 \%)$ & \\
Spasticity & & & \\
$-\quad$ MAS 1 & $3 / 5(60 \%)$ & $1 / 5(20 \%)$ & 0.221 \\
$-\quad$ MAS 2 & $2 / 5(40 \%)$ & $4 / 5(80 \%)$ & \\
\hline
\end{tabular}

${ }^{\mathrm{a}}$ Independent sample t-test, ${ }^{\mathrm{b}}$ Fisher's exact test, ${ }^{\mathrm{c}}$ Mann Whitney test;

The authors analyzed the possible influence of the mean initial RMS value on the research results. The mean initial RMS value in the treatment group it was $0.123 \pm 0.099 \mathrm{mV}$, while in the control group was 0.209 $\pm 0.098 \mathrm{mV}$. The Shapiro Wilk normality test showed the initial and final values of the mean RMS values in both groups were normally distributed. The results of the Independent Sample T-Test showed the mean initial RMS value between the two groups was not different $(p=0.207)$, so it was not expected to affect the results of the study.

The results of this study showed that there were no significant differences in wrist extensor muscle activity before and after the intervention in the control group $(p=0.446)$, the treatment group $(p=0.764)$, or between the two groups $(p=0.917)$. Cohen's d mean effect size analysis showed a medium result (Cohen's $d=0.59$ ). The RMS value of the wrist extensor muscles is shown in table 2, while the box and whisker diagram of the average RMS before and after the intervention is shown in figure 1.

Table 2. RMS values before and after intervention

\begin{tabular}{lccccccc}
\hline \multirow{2}{*}{$\begin{array}{c}\text { RMS wrist extensor } \\
\text { activity }(\mathrm{mV})\end{array}$} & \multicolumn{2}{c}{ EG $(\mathrm{n}=5)$} & $\begin{array}{c}\text { Within- } \\
\text { group EG }\end{array}$ & CG $(\mathrm{n}=5)$ & \multicolumn{2}{c}{$\begin{array}{c}\text { Within- } \\
\text { group CG }\end{array}$} & Between group \\
\cline { 2 - 8 } & Pre & Post & $p$ value & Pre & Post & $p$ value & $p$ value \\
\hline Mean & 0.123 & 0.134 & 0.446 & 0.209 & 0.215 & 0.764 & 0.917 \\
SD & 0.099 & 0.111 & 0.098 & 0.131 & & 0.764 \\
\hline
\end{tabular}

* Significant at $\mathrm{p}<0.05$ 


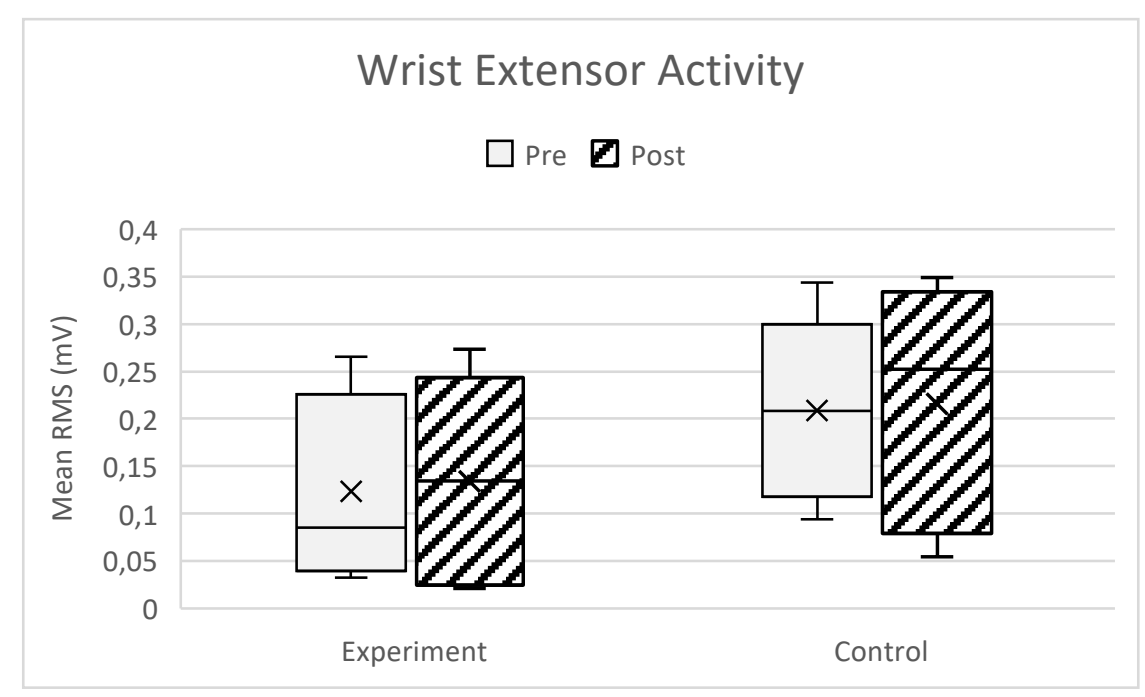

Fig 1. Box and whisker diagram for RMS values before and after intervention

\section{Discussion}

This study showed that the implementation of 5 anodal tDCS sessions had no effect on changes in wrist extensor muscle activity $(\mathrm{p}=0.917)$. The results of this study were different from the research conducted by Shaheiwola et al. (2018). That study compared the effect of adding tDCS therapy to functional electrical stimulation (FES) on improving upper extremity function in 30 subjects with chronic post-stroke patients, with one of the outcome parameters being sEMG evaluation to assess muscle activation. The subjects were divided into two groups, the intervention group (active tDCS and FES) and the control group (sham tDCS and FES). Subjects received therapy five times per week for four weeks. Active bilateral tDCS was administered with $5 \times 5 \mathrm{~cm}$ electrodes, $2 \mathrm{~mA}$ intensity, for 20 minutes. In the experimental group, activity of the anterior deltoid muscle, extensor carpi radialis longus, and flexor digitorum superficialis increased significantly (Shaheiwola et al., 2018). The results of this study may differ due to variances in the tDCS parameters and intervention period.

The lectrode size, intensity, and duration of tDCS therapy in this study and that of Shaheiwola et al. (2018) were no different. The difference in tDCS parameters lies in the application technique, where Shaheiwola et al. (2018) used bilateral tDCS, while this study used A-tDCS. Research related to the different effects of tDCS application techniques (A-tDCS, C-tDCS, and bilateral tDCS) on upper extremity function in post-stroke patients has been previously conducted by Mahmoudi et al. (2011) and Fusco et al. (2013). Both studies showed that the three tDCS application techniques have a significant effect compared to sham application. Study results from Mahmoudi et al. (2011) showed the highest improvement in the bilateral tDCS group. In contrast, study results from Fusco et al. (2013) showed A-tDCS resulted in higher hand dexterity improvements than others (Fusco et al., 2013). Sehm et al. (2013) examined the changes in interhemispheric connectivity in healthy people who were given tDCS therapy. The study used functional MRI (fMRI) to compare the effects of bilateral and unilateral tDCS on motor cortex connectivity. The results of that study indicate that both bilateral and unilateral tDCS cause changes in interhemispheric connectivity, where the changes that occurred were not significantly different between the two techniques (Sehm et al., 2013). 
The interhemispheric inhibitory balance is disturbed after a stroke event. Improvement of interhemispheric inhibitory balance disorders is one of the basic working mechanisms of tDCS in post-stroke rehabilitation, which is done by either increasing the excitability of the lesioned motor cortex (with A-tDCS) or decreasing the excitability of the healthy motor cortex (with C-tDCS), or both (with bilateral tDCS) (Hummel and Cohen, 2006; Schlaug et al., 2008). Anodal tDCS causes nerve membrane depolarization which then increases calcium influx, causing after-effects through a long-term potentiation mechanism (Nitsche and Paulus, 2001; Rozisky et al., 2015). This study used anodal tDCS since a meta-analysis study found that using tDCS with anodal stimulation to the lesioned M1 was significantly advantageous for the restoration of upper extremity motor function in stroke patients (Butler et al., 2013).

The study intervention period of Shaheiwola et al. (2018) was for 4 weeks (20 sessions), while this study was for one week (5 sessions). Increased intensive stroke rehabilitation therapy could improve functional recovery, but the benefits of the intensity of therapy may not be uniform or proportional (Teasell and Hussein, 2018). To the authors' knowledge, there is no recommendation on the best duration of intervention with tDCS for post-stroke patients. Several days of tDCS stimulation sessions are generally performed to produce significant clinical effects, which involves five stimulation sessions (on five consecutive days) or ten sessions (on ten days out of 14 possible days). It should be noted that the choices were based more on feasibility rather than evidence of a difference in efficacy between one stimulation period over the other (Hamilton et al., 2019). In terms of occupational therapy, Galvin et al. (2008) discovered that increasing the duration of therapy had a minor but significant effect on increasing the ability to do activities of daily living after a stroke, but not on hand function. The difference between the results of this study and of Shaheiwola et al. (2018) may indicate that the five-day intervention period may not be sufficient to lead to improvements in wrist extension control, which is one of the most difficult movements to achieve recovery after a stroke event (Cauraugh et al., 2000).

Interestingly, we found that muscle strength and spasticity possessed a significant correlation with changes in wrist extensor activity. The wrist extensor muscles are important for hand dexterity and stabilization during grip and pinch activities. After a stroke, wrist extension control is one of the primary causes of hand dysfunction and one of the most difficult movements to achieve recovery (Cauraugh et al., 2000; Souza et al., 2017). In this study, we discovered that muscle strength has a strong positive correlation with delta RMS value $(r=0.790, p=0.007)$, while spasticity has strong negative correlation $(r=-0.711, p=0.021)$. After a stroke, motor unit control of the affected muscles may be lost (weakness) and voluntary muscle activation changes cause co-contraction of antagonist muscles and decreased activation of agonist muscles (Chae et al., 2002). Spasticity acts as a limiting factor to achieving upper extremity motor and functional recovery (Cacho et al., 2017). However, there were difficulties measuring the definite influence of spasticity on motor impairment after a stroke, as the level of spasticity may differ according to the task demands (Sommerfeld et al., 2004).

There were several study limitations which we encountered during this study. These consisted of limited sample size, absence of blinding, and assessment of the thickness of subcutaneous fat tissue which could affect sEMG signaling was not performed. Furthermore, neurophysiological measurements such as motorevoked potentials and intracortical inhibition and facilitation were not assessed.

Generalization of the results of this study into the population requires caution due to the limited number of subjects. Studies have shown a beneficial effect of tDCS on upper extremity recovery after a stroke event (Cha et al., 2014; Butler et al., 2013). A study carried out by Cleland et al., (2020) showed the use of tDCS in an outpatient clinical setting is feasible and safe. Research on the effect of tDCS on UE function in poststroke patients is a relatively new study in Indonesia. The authors hope there will be further studies conducted with a larger number of subjects and a longer intervention period regarding the effects of tDCS on muscle activity after a stroke. 


\section{Conclusion}

There were no significant effects after five sessions of anodal tDCS on wrist extensor activation in poststroke patients. Further studies with a larger sample size and a longer period of intervention are necessary to clarify the effects of tDCS on wrist extension control in stroke patients.

\section{Conflict of Interests}

The authors declare that there is no conflict of interests regarding the publication of this paper.

\section{Acknowledgements}

The authors would like to thank Amandha Boy Timor, MD and the late Budiono, MD for their assistance with statistical analysis.

\section{References}

Barreca S, Wolf SL, Fasoli S, Bohannon R. (2003). Treatment interventions for the paretic upper limb of stroke survivors: a critical review. Neurorehabil Neural Repair. 2003;17(4):220-226

Butler AJ, Shuster M, O’Hara E, Hurley K, Middlebrooks D, Guilkey K. (2013). A meta-analysis of the efficacy of anodal transcranial direct current stimulation for upper limb motor recovery in stroke survivors. Journal of Hand Therapy 26;162-171

Cacho R, Cacho E, Loureiro AB, Cirne G, Pereira SA, Pegado R, Lima N, Borges G. (2017). The spasticity in the motor and functional disability in adults with post-stroke hemiparetic. Fisioterapia em Movimento. 30. 745-752. 10.1590/1980-5918.030.004.ao09.

Cauraugh J, Light K, Kim S, Thigpen M, Behrman A. (2000). Chronic motor dysfunction after stroke: recovering wrist and finger extension by electromyography-triggered neuromuscular stimulation. Stroke. 31(6):1360-4.

Chae, J., Yang, G., Park, B. K., \& Labatia, I. (2002). Muscle Weakness and Cocontraction in Upper Limb Hemiparesis: Relationship to Motor Impairment and Physical Disability. Neurorehabilitation and Neural Repair 2002;16:241-248.

Cleland BT, Galick M, Huckstep A, Lenhart L, Madhavan S. (2020). Feasibility and Safety of Transcranial Direct Current Stimulation in an Outpatient Rehabilitation Setting After Stroke. Brain Sciences. 2020; 10(10):719. https://doi.org/10.3390/brainsci10100719

Cram JR, Kasman GS, Holtz, J. (2011). Atlas For Electrode Placement dalam Criswell E., et al. Cram's Introduction To Surface Electromyography $2^{\text {nd }}$ Ed. Boston, MA: Jones And Bartlett Publishers

Feigin FL, Norrving B, Mensah GA. (2017). Global burden of stroke. Circ Res. 120:439-448. doi:10.1161/circresaha.116.308413

Fusco, A., De Angelis, D., Morone, G., Maglione, L., Paolucci, T., Bragoni, M., \& Venturiero, V. (2013). The ABC of tDCS: Effects of Anodal, Bilateral and Cathodal Montages of Transcranial Direct Current Stimulation in Patients with Stroke-A Pilot Study. Stroke Research and Treatment, 2013, 1-6. https://doi.org/10.1155/2013/837595

Galvin, R., Murphy, B., Cusack, T., \& Stokes, E. (2008). The Impact of Increased Duration of Exercise Therapy on Functional Recovery Following Stroke-What Is the Evidence? Topics in Stroke Rehabilitation, 15(4), 365-377. https://doi.org/10.1310/tsr1504-365

Hatem SM, Saussez G, della Faille M, Prist V, Zhang X, Dispa D, Bleyenheuft Y. (2016). Rehabilitation of Motor Function after Stroke: A Multiple Systematic Review Focused on Techniques to Stimulate Upper Extremity Recovery. Front. Hum. Neurosci. 10:442. doi: 10.3389/fnhum.2016.00442

Hermens HJ, Freriks B, Disselhorst-Klug C, Rau G. (2000). Development of recommendations for SEMG sensors and sensor placement procedures. Journal of Electromyography and Kinesiology 10 (2000) 361-374

Hummel FC dan Cohen LG. (2006). Non-Invasive brain stimulation: a new strategy to improve neurorehabilitation after stroke? LancetNeurol. 5,708-712. doi:10.1016/S1474-4422(06)70525-7

Hunter SM dan Crome P. (2002). Hand function and stroke. Reviews in Clinical Gerontology, 12, pp 68-81. doi:10.1017/S0959259802012194

Klomjai W, Lackmy-Valle A, Roche N, Pradat-Diehl P, Marchand-Pauvert V, Katz R. (2015). Repetitive transcranial magnetic stimulation and transcranial direct current stimulation in motor rehabilitation after stroke: An update. Ann Phys Rehabil Med. http://dx.doi.org/10.1016/ j.rehab.2015.05.006

Lee SA, Lee SH, Jung BK. (2015). Analysis of cortical activation during three types of therapeutic activity. J Phys Ther Sci. 2015 Apr;27(4):1219-22. doi: 10.1589/jpts.27.1219

Mahmoudi, H., Haghighi, A. B., Petramfar, P., Jahanshahi, S., Salehi, Z., \& Fregni, F. (2011). Transcranial direct current stimulation: Electrode montage in stroke. Disability and Rehabilitation, 33(15-16), 1383-1388. https://doi.org/10.3109/09638288.2010.532283

Moore KL, Dalley AF, Agur AMR. (2010). “Upper Limb” in Moore et al. Clinically Oriented Anatomy (6th ed.) p750-3. Philadelphia: 
Lippincott Williams \& Wilkins

Neumann. (2010). "Wrist" in Neumann et al. Kinesiology of the musculoskeletal system: foundations for rehabilitation p235-8. St. Louis :Mosby.

Nitsche MA dan Paulus W. (2001). Sustained excitability elevations induced by transcranial direct current motor cortex stimulation in humans. Neurology; 57,1899-1901. doi:10.1212/WNL.57.10.1899

Rozisky JR, Antunes LC, Brietzke AP, de Sousa AC, Caumo W. (2015). Transcranial direct current stimulation and neuroplasticity in: Rogers L. Transcranial Direct Current Stimulation (tDCS): Emerging Uses, Safety And Neurobiological Effects p63-75. Nova Pub Inc. https://www .researchgate.net/publication/305439421

Schlaug G, Renga V, Nair D. (2008). Transcranial direct current stimulation in stroke recovery. Arch Neurol. 2008;65(12):1571-1576. doi:10.1001/archneur.65.12.1571.

Sehm, B., Kipping, J., Schäfer, A., Villringer, A., \& Ragert, P. (2013). A Comparison between Uni- and Bilateral tDCS Effects on Functional Connectivity of the Human Motor Cortex. Frontiers in Human Neuroscience, 7. https://doi.org/10.3389/fnhum.2013.00183

Shaheiwola, N., Zhang, B., Jia, J., \& Zhang, D. (2018). Using tDCS as an Add-On Treatment Prior to FES Therapy in Improving Upper Limb Function in Severe Chronic Stroke Patients: A Randomized Controlled Study. Frontiers in Human Neuroscience, 12(June), 1-11. https://doi.org/10.3389/fnhum.2018.00233

Sommerfeld DK, Eek EU, Svensson AK, Holmqvist LW, von Arbin MH. (2004). Spasticity after stroke: its occurrence and association with motor impairments and activity limitations. Stroke. 2004 Jan;35(1):134-9. doi: 10.1161/01.STR.0000105386.05173.5E.

Souza, V. K., Claudino, A. F., Kuriki, H. U., Marcolino, A. M., Fonseca, M. de C. R., \& Barbosa, R. I. (2017). Fatigue of the wrist extensor muscles decreases palmar grip strength. Fisioterapia e Pesquisa, 24(1), 100-106. https://doi.org/10.1590/18092950/17328524012017

Strong, K., Mathers, C., and Bonita, R. (2007). Preventing stroke: saving lives around the world. Lancet Neurol. 6, 182-187. doi: 10.1016/S1474-4422(07)70031 